\title{
EDITORIAL
}

Kritik an, in und durch Kommunikations- und Medienwissenschaft Editorial zum Sonderheft

Criticism of, in, and through communication and media studies Editorial to the Special Issue

Peter Gentzel, Sigrid Kannengießer, Cornelia Wallner \& Jeffrey Wimmer 
Peter Gentzel (Jun.-Prof. Dr.), Institut für Buchwissenschaften, Friedrich-Alexander-Universität Erlangen-Nürnberg, Katholischer Kirchenplatz 9, 91054 Erlangen, Germany. Contact: Contact: peter.gentzel(at)fau.de. ORCID: https://orcid.org/oooo-0002-3407-8231

Sigrid Kannengießer (Prof. Dr.), Zentrum für Medien-, Kommunikations- und Informationsforschung, Universität Bremen, Linzer Str. 4, 28359 Bremen, Contact: sigrid.kannengiesser (at)uni-bremen.de, https://orcid.org/oooo-0002-2342-9868

Cornelia Wallner (Dr.), Institut für Kommunikationswissenschaft und Medienforschung, Ludwig-Maximilians-Universität München, Oettingenstr. 67, 80538 München, Germany. Contact: cornelia.wallner(at)ifkw.Imu.de. ORCID: https://orcid.org/oooo-0003-2799-0182

Jeffrey Wimmer (Prof. Dr.), Institut für Medien, Wissen und Kommunikation, Universität Augsburg, Universitätsstr. 10, 86135 Augsburg, Germany. Contact: jeffrey. wimmer(at)phil.uni-augsburg.de 


\title{
EDITORIAL
}

\section{Kritik an, in und durch Kommunikations- und Medienwissenschaft Editorial zum Sonderheft}

\author{
Criticism of, in, and through Communication and Media Studies \\ Editorial to the Special Issue
}

\author{
Peter Gentzel, Sigrid Kannengießer, Cornelia Wallner \& Jeffrey Wimmer
}

Zusammenfassung: Vor dem Hintergrund des Bedeutungsverlusts kommunikationswissenschaftlicher Perspektiven in der Öffentlichkeit und der gegenwärtigen gesellschaftlichen Herausforderungen setzt sich das Sonderheft mit der Breite der Bedeutung von Kritik in der Kommunikationswissenschaft auseinander: mit Kritik an, in und durch die Kommunikationswissenschaft. Einleitend werden vier zentrale Dimensionen von Kritik hinsichtlich Theorien, Methoden, Forschungsgegenständen und dem disziplinären Selbstverständnis der Kommunikationswissenschaft skizziert. Es wird deutlich, dass in einer digital transformierten Welt die Kommunikationswissenschaft auf die Entwicklung und damit den Wandel ihrer theoretischen wie methodischen Instrumente angewiesen ist, um angemessen verstehen und erklären zu können. Daran anknüpfend thematisieren und analysieren die Beiträge des Sonderhefts verschiedene relevante Aspekte und Fallbeispiele. Sie verdeutlichen aber auch weiterführende Fragen und Forschungsdesiderate.

Schlagwörter: Digitalisierung, Kommunikationswissenschaft, Medienkritik, Methodologie, Theorien, Wissenschaftsverständnis.

\begin{abstract}
Against the background of the loss of importance of communication and media studies in public and the current social challenges, the special issue deals with the breadth of the meaning of criticism in communication science: with criticism of, in and through communication and media studies. Initially, four central dimensions of criticism with regard to theories, methods, research objects and the disciplinary identity of communication and media studies are outlined. It becomes clear that in a digitally transformed world, communication and media studies is dependent on the development and thus the change of theoretical and methodological instruments in order to be able to understand and explain appropriately. Following on from this, the articles in the special issue address and analyze different relevant aspects and case studies. But they also clarify further questions and research desiderata.
\end{abstract}

Keywords: Digitalization, communication and media studies, media criticism, methodology, theories, understanding of science. 


\section{Ausgangsbeobachtungen}

Gesellschaftliche Funktion und Legitimität (freier) sozialwissenschaftlicher Forschung ist zweifelsohne an ihre Fähigkeit zur Kritik an gesellschaftlichen Phänomenen und Prozessen gebunden. Daher gehört es zum Selbstverständnis kommunikationswissenschaftlicher Forschung, medienvermittelte, durch Medien konstituierte und auf Medien bezogene kommunikative Praktiken, Diskurse und Öffentlichkeiten theoretisch zu begreifen und kritisch zu analysieren. Konstitutiv für gelungene Kritik ist dabei immer das Beurteilen des Gegebenen und das Aufzeigen von Alternativen, wofür ganz unterschiedliche Wege offenstehen: die historische Rekonstruktion, das theoretische Hinterfragen, Einordnen und Bewerten, und die vergleichende empirische Analyse. Schließlich verändern sich Form, Legitimierung und Gegenstand der Kritik selbst im Rahmen sozialer und kultureller, ökonomischer und politischer sowie kommunikativer und medialer Entwicklungen.

Für die Kommunikationswissenschaft der Gegenwart ist Kritik in mindestens drei Dimensionen relevant: Zum einen ist Kritik als Medienkritik und bezogen auf medienkritische Praktiken Gegenstand theoretischer und empirischer Forschung. Zum anderen nehmen Kommunikationswissenschaftler*innen kritische Perspektiven auf mediatisierte Phänomene, Diskurse und Praktiken in ihren Theorien und empirischen Studien ein. Schließlich ist die Kommunikationswissenschaft selbst kritisch in den Blick zu nehmen: nicht nur ihre Theorien und Perspektiven, sondern auch die Methoden der Kommunikations- und Medienforschung, welche in Hinblick auf ihre Relevanz, aber auch auf ihre Bedeutung für die Erkenntnismöglichkeiten der Forschung zu prüfen sind.

Trotz der Relevanz von Kritik in der Kommunikationswissenschaft nehmen wir einen Bedeutungsverlust sozial- und insbesondere kommunikationswissenschaftlicher Kritik in gesellschaftlichen und wissenschaftlichen Diskursen wahr. So hält sich eine überwiegend positivistisch geprägte, eher auf quantitative Methoden gestützten Forschung mit der grundlegenden Kritik an den Formalobjekten zurück. Es ist es die Kritik durch kommunikationswissenschaftliche Forschung, die dem Puls gesellschaftspolitischer Veränderungen nur schwer folgen kann und am Kern drängender gesellschaftlicher Debatten vorbei geht oder aufgrund ihrer Ideen- und Sozialgestalt aktuell vorbeigehen muss.

Vor dem Hintergrund dieses Bedeutungsverlusts bzw. der gesellschaftlichen Herausforderungen haben wir für die Fachgruppe Soziologie der Medienkommunikation der Deutschen Gesellschaft für Publizistik und Kommunikationswissenschaft eine Tagung an der Universität Augsburg 2019 ausgerichtet, deren Vorträge sich mit der Breite der Bedeutung von Kritik in der Kommunikationswissenschaft auseinandersetzten: mit Kritik an, in und durch die Kommunikationswissenschaft. Die in diesem Sonderheft versammelten Beiträge basieren alle auf Präsentationen dieser Konferenz. Sie setzen sich (kritisch) mit theoretischen Ansätzen zum Kritikbegriff auseinander (Adolf), mit Kritik als Forschungsgegenstand (Kannengießer \& Möller), sie üben Kritik an den Methoden der Kommunikationswissenschaft (Fürst) und kritisieren das Fach der Kommunikationswissenschaft - auch in Hinblick auf den Bedeutungsverlust von Kritik (Altmeppen und Krotz). Im Angesicht von Qualität und Quantität der zusammengetragenen Stimmen beschränken wir 
uns, dieses Themenheft einleitend, auf jeweils kurze, selektive Schlaglichter in den vier Dimensionen, die von den Beiträgen der vorliegenden SCM vertieft diskutiert werden: Theorie, Methode, Materialobjekt und Sozialgestalt der disziplinären Forschung.

\section{Kritik aus theoretischer Perspektive}

Aus theoretischer Sicht wollen wir die Aufmerksamkeit auf zwei Prozesse lenken, die, obwohl auf unterschiedliche Phänomene zielend und in deutlich unterscheidbaren Diskursen verhandelt, Bedeutung, Form und Leistungsvermögen sozialwissenschaftlicher Kritik in der Gegenwart grundlegend in Frage stellen: Datafizierung und die sozial-/kulturtheoretische Bedeutung der Profilierung flacher Ontologien in den letzten zwei Jahrzehnten. Es geht also in theoretischer Hinsicht um die epistemischen Fragen nach der Bedeutung digitaler Daten als analytisches Material, als Bausteinen sozialwissenschaftlicher Kritik einerseits und den Konsequenzen flacher oder symmetrischer Ontologien für Wesen und Leistungsvermögen der Kritik andererseits. Wenn auch aus unterschiedlichen Gründen stimmen die Einschätzungen aus diesen beiden Diskursen in letzter Konsequenz darin überein, dass Kritik, die sich zentral auf klassische theoretische Konzepte (z. B. des Subjekts, der Struktur, Handlung oder Entfremdung) stützt, keinen Wert mehr hat.

Die eine Seite argumentiert, dass sich im Zuge von Datafizierung die Limitationen statistischer Forschung aufgelöst haben, weil Menge und Dichte der erhobenen Daten sowie deren automatische Verarbeitung ein Niveau erreicht haben, die Fragen der Auswahl und des Samplings, der Stichprobengröße und deren Berechenbarkeit obsolet machen. Insofern diese Grenzen gefallen sind, heben Prozesse der Datafizierung das Bestreben der Menschheit nach Verständnis der Welt auf eine neue Stufe. Bereits die erste sozialwissenschaftliche Definition von Datafizierung aus dem Jahr 2013 beschreibt das Phänomen als „activity“ of „taking all aspects of life and turning them into data [... as] latest step in humanity's quest to understand and quantify the world" (Cukier \& Mayer-Schoenberger, 2013, S. 34) - und postuliert damit die Identität von Verstehen und Quantifizierung sowie die Legitimität von Weltverständnis und -erklärung mittels und durch digitale Datenverarbeitung. Wenn alle Aspekte der Welt und des Handelns - Zeit, Raum, Sozialität, Bedeutung - in Form von Daten vorliegen, müsse sich folgerichtig auch das Betriebsprogramm der Sozialwissenschaften von der Hermeneutik zur Korrelation wandeln, d. h. von der Auslegung und Argumentation zur Mustererkennung und (selbstevidenten) Visualisierung. Theorie und damit auch theoretisch geleitete Kritik ist in dieser Argumentation absolut folgerichtig dann auch hinfällig (u. a. Anderson, 2008; Savage, 2015).

Auf der anderen Seite steht ein Argumentationsmuster, das in der Entwicklung des sozial- und kulturtheoretischen Diskurses, sei es unter dem Signet des „New Materialism“ (Kallmeyer, 2017), des „Practice Turn“ (Schatzki, 2001) oder der „Akteur-Netzwerk-Theorie“ (Latour, 2004), seit der Jahrtausendwende vielfach vorgebracht wurde: Im Angesicht einer zunehmend technologisch durchdrungenen, digital transformierten Welt ist es demnach nicht weiter sinnvoll, distinkt zwischen Subjekten und Objekten zu unterschieden, ist die Produktion von Wissen nicht 
mehr nur allein dem technische Geräte bedienenden Mensch zuzuschreiben, ist Handeln nicht mehr exklusives Privileg des Individuums. All diese ex ante gesetzten und ideengeschichtlich tradierten Kategorien würden ein adäquates Verstehen, den wahren Blick auf die Welt verdecken, da diese allein aus Relationen, Assoziationen und Assemblagen besteht, in der Qualitätszuschreibung wie Subjekt und Objekt fragwürdig scheinen, Wissensgenerierung und Handlungsmacht eine zwischen Menschen, Artefakten, Routinen und Protokollen verteilte Angelegenheit ist. Die benannten theoretischen Strömungen oder „turns“ stimmen sicherlich nicht in jedem Aspekt überein, ontologisch zeichnen sie sich insgesamt jedoch durch die Neubestimmung des Verhältnisses von menschlichen Subjekten und technologischen oder natürlichen Objekten auf ein "flaches“ oder "symmetrisches“ Level aus. Folglich sind auch in diesem Diskurs Stimmen zu vernehmen, die der traditionellen, auf ex ante definierten Kategorien basierenden Kritik zunehmenden Bedeutungsverlust bescheinigen (Latour, 2004).

Die Kommunikationswissenschaft versteht sich als sozialwissenschaftliche Disziplin, deren ideengeschichtliche Wurzeln eng mit der Soziologie Max Webers verbunden sind. In epistemologischer Hinsicht wollen wir deshalb zwei Gedanken Max Webers zum Ausgangspunkt für die weitere Einordnung und Befragung der soeben skizzierten Argumentationszusammenhänge nehmen: Weber umreißt das Arbeitsprogramm der verstehenden Soziologie mit dem Auftrag „soziales Handeln deutend [zu] verstehen und dadurch in seinem Ablauf und seinen Wirkungen ursächlich [zu] erklären“ (Weber, 1988 [1922], S. 1). Auf diesen Satz gehen zwei für die Kommunikationswissenschaft zentrale, paradigmatische Weichenstellungen zurück. Dies sind in methodologischer Hinsicht die Präzisierung und Unterscheidung von Analyselogiken in deutendes Verstehen und ursächliches Erklären. Theoretisch wegweisend ist weiterhin der Begriff des sozialen Handelns, der die Abgrenzung zu bloßem Verhalten ermöglicht, indem er auf den subjektiven (Handlung) und sozialen (soziales Handeln) Sinn fokussiert. Die Kommunikationswissenschaft nutzte in der Folge genau diesen Sinnbegriff paradigmatisch, um ihren zentralen Erkenntnisgegenstand Kommunikation von mathematisch-technischen Modellen oder psychologisch-behavioristischen Vorstellungen (etwa Watzlawicks „Man kann nicht nicht kommunizieren. “) abzugrenzen (u. a. Burkart, 2002; Krallmann \& Ziemann, 2001; Krotz, 2008). Zudem wird Kommunikation durch die Bindung an sozialen Sinn zu einem Phänomen, das untrennbar mit Sozialität und Gesellschaft sowie Wissen und Kultur verwoben ist. Die Frage, was die Disziplin unter Kommunikation versteht, wird also im ersten Schritt mit dem Verweis auf Sinn und die daran gekoppelten Prozesse der Sinngebung und des Sinnverstehens beantwortet, die Frage wie man dies tut mit dem Verweis auf methodisch geleitetes Verstehen und Erklären. Ohne jeden Zweifel haben sich die Formen der Sinngebung und des Sinnverstehens sowie der Methoden zu deren Erforschung in den beinahe 100 Jahren seit Webers Veröffentlichung verändert, haben sich Fragen und Perspektiven der Disziplin deutlich ausdifferenziert. Das Verstehen und Erklären sozialen Sinns als disziplinärer, epistemologischer Anker ist in die Einführungswerke und -vorlesungen der Kommunikationswissenschaft, in theoretische wie analytische Handbücher aber weiterhin fest eingeschrieben (z. B. Burkart, 2002, S. 20-24; Krallmann \& Ziemann, 2001, S. 155; Pürer 2003, S. 59;). Es gibt also gute Gründe, die epis- 
temologische Position der Disziplin mit Max Weber zu konturieren und nun weiter zu nutzen, um beispielhaft den Wert digitaler Daten für das Verstehen von Sinn einzuordnen sowie den Stellenwert von Sinngebung und Sinnverstehen im Kontext symmetrischer Ontologien zu beleuchten.

Digitale Daten, deren Erfassung und automatische Verarbeitung erheben, wie wir gezeigt haben, den Anspruch auf Weltverstehen und -erklärung - allerdings in einem ganz anderen Sinne als es Max Weber formuliert hat. Chris Anderson, Chefredakteur des Wired Magazins, hat das auf digitalen Daten basierte Erkenntnismodell pointiert zusammengefasst:

„Forget taxonomy, ontology, and psychology. Who knows why people do what they do? The point is they do it, and we can track and measure it with unprecedented fidelity. With enough data, the numbers speak for themselves." (Anderson, 2008)

Das von Anderson proklamierte - und nebenbei im Dienste einer kapitalistischen Verwertungslogik stehende - Erkenntnismodell ist mit sozialwissenschaftlich-kritischem Verstehen von Welt nicht vereinbar, nicht zuletzt weil der ihnen inhärente, singuläre Wahrheits- und Objektivitätsanspruch ein grundlegend anderer ist und weder mit den sich diskursiv zu behauptenden Modellen des Erklärens im Sinne Karl Poppers noch jenen des Verstehens von Theodor Adorno vereinbar ist (siehe hierzu der Beitrag von Marian Adolf in diesem Sonderheft). Die Aufgabe der kommunikationswissenschaftlichen Kritik geht über diese Diskussion allerdings noch hinaus und hat sich gegenüber der klar formulierten Aufgabe des epistemologischen Eckpfeilers „Sinnverstehen“ zu positionieren. Dem Chor der allgemeineren geistesund sozialwissenschaftliche Kritik am mathematisch-naturwissenschaftlichen Erkenntnismodell der computational data science (u. a. Manovich, 2020) muss sie einen wichtigen Aspekt hinzufügen: Kommunikation, Medienaneignung und -nutzung meinen etwas anderes als aus Daten ablesbare Verhaltensmuster. Mediennutzung ist mit soziokulturellen Zeitordnungen auf eine Weise verbunden, die sich nicht allein mit gemessenem Zeitpunkt und quantifizierter Dauer verstehen lässt (Görland, 2020; Neverla, 1992); die Konstitution von Kommunikationsräumen umfasst mehr als Orte georeferenzierter Mediennutzung (Wallner \& Adolf, 2014); kommunikative Bedeutung leitet sich nicht allein aus Medieninhalten ab und Sinnverstehen ist nicht wahr oder falsch - zudem beides ohne die Berücksichtigung gesellschaftlicher Machtstrukturen nicht zu fassen ist (Hall, 1997). Der Besitz von und die Macht über Daten liegt in der Gegenwart bei einigen wenigen Akteur*innen, die von einem behavioristischen Verständnis von Kommunikation durchdrungen sind und ihre Produkte, Medientechnologien und Kommunikationsplattformen durch Verhaltensvorhersage und -manipulation ökonomisch verwerten (Zuboff, 2019, S. 292-326). Kommunikationswissenschaftliche Kritik wird mit rein datenbasierten Erkenntnismodellen in dem skizzierten Sinne nicht in Konkurrenz treten können - allein schon deshalb, weil es die mathematisch-naturwissenschaftliche Wahrheit nur im Singular gibt. Sie verfügt aber über alternative, plurale Ansätze des Verstehens, Erklärens und Deutens von Kommunikationsprozessen, die für unterschiedliche Menschen an verschiedenen Orten zu differenten Zeitpunkten, 
für gesellschaftliche Entwicklungen und die Aushandlung kulturellen Wissens (im Plural) wertvoll sind - und sollte diese auch entsprechend verteidigen.

Die Frage nach den Mitteln für ein solches Unterfangen führt unweigerlich zu Begriffen und Theorien, d.h. den grundlegenden Annahmen und Perspektiven, die jeden Verstehens- und Erklärungsbemühungen vorausgehen. Wir haben in diesem Zusammenhang auf verschiedene Strömungen hingewiesen, die in dem Postulat flacher oder symmetrischer Ontologien übereinstimmen. Es ist offenkundig, dass eine sich wandelnde, insbesondere digital transformierte Welt auf die Entwicklung und damit ebenfalls den Wandel der Instrumente - im Falle der Theorie also Begriffe und Konzepte - angewiesen ist, um verstanden und erklärt werden zu können. Die Stoßrichtung der skizzierten theoretischen Veränderungen ist also vollkommen nachvollziehbar und richtig: Wenn die Konstitution, Veränderung und Reproduktion sozialer Praktiken und Phänomene, wenn Zugang, Verteilung und Aneignung kulturellen Wissens basal mit Artefakten verbunden sind und über kapitalistisch organisierte und bewährte Technologien erfolgen, dann ist die Bedeutung technischer Artefakte als bloße Mittel im Dienst intentionaler Handlungen autonomer Individuen nicht adäquat. Akteur-Netzwerke und Assemblagen sind theoretische Konzepte, die zunehmend komplexe Prozesse zu verstehen suchen, indem sie traditionell bemühte, verfestigte Kategorien aufbrechen und neuen „Akteure*inen“ Macht zuschreiben. In diesem Sinne ist der oben zitierte Bedeutungsverlust der klassischen Kritik zu verstehen und die These auch nachzuvollziehen. Auch an dieser Stelle sei aber die Frage nach Rolle und Bedeutung des Sinnverstehens im Anschluss an Max Weber gestellt. Nick Couldry hat jüngst auf die Homologie datenbasierter Welterklärungen und dem „radikalen Reduktionismus“ bzw. „descriptivism“ der AkteurNetzwerk-Theorie hingewiesen und gezeigt, dass alleine mit den Mitteln flacher Ontologien einer mustererkennenden Verhaltensanalyse und dem daraus resultierenden mathematischen Weltverstehen kein kraftvoller Widerspruch entgegenzusetzen ist (Couldry, 2020). Couldry erläutert dieses Argument mit Blick auf die gesellschaftliche Dimension und relationale Konzepte von Macht und Ordnung. Hinzufügen möchten wir hierzu die Aufgabe einer kritischen Weiterentwicklung so grundlegender Begriffe wie Subjektivität, Weltbild oder Entfremdung. Möglicherweise ist der Weg von der ontologisch flachen Position zur sozialwissenschaftlichen Verabschiedung des Subjekts keine Einbahnstraße (und hat seinen größten Wert in der Kritik und am Überwinden des erkenntnistheoretischen Dualismus). Unter Umständen finden sich auch Möglichkeiten, subjektives Sinnverstehen und menschliche Handlungen genealogisch zu verstehen und mittels Assemblagen und (auch teilweise desorganisierten, ungeordneten) Netzwerken zu erklären statt aufzugeben. Auch der Versuch, Handlungserklärungen von der rationalen Intention des Subjekts zu lösen, ohne die Kategorie des Weltbezugs aufzugeben und damit die Qualität der Reflexion oder Sorge zu negieren, ist nicht zum Scheitern verurteilt (Kalthoff, 2019). Auf diese Weise ließe sich nämlich eine Grundbedingung der Kritik erhalten: die Überschreitung des Gegebenen - unter Umständen sogar im Dienste des Gewünschten. Anschluss fände man auf diese Weise auch an leistungsstarke kritische Konzepte wie etwa jenes der Entfremdung (Gentzel, 2019). Wenn die philosophischen Nestoren der flachen Ontologien zur Fundierung von Praktiken, Netzwerken und Assemblagen bemüht werden (u. a. Schatzki et al., 2001), 
dann ließe sich - etwa als Pendant zu Couldrys Bezug auf Macht und Ordnung sicher auch an deren stärker kulturanalytisch ausgerichtete Arbeiten anknüpfen. Beispielsweise indem man sozialwissenschaftlich das Wesen der Technik als „ganz und gar nichts Technisches" (Heidegger, 2000, S. 9) begreift und analysiert, welche Art Weltbilder mit digitalen Technologien verknüpft sind und in welchem Zusammenhang diese mit Kreativität und Spontaneität oder einer bloßen „Versiegelung“ von Lebenswelt stehen (Luckner, 2008). Der in dieser Hinsicht erste Schritt einer kritischen Analyse von Werten, Visionen und Weltbildern die Gestaltung und Design von Technologien und Prozessen der Digitalisierung und Datafizierung zu Grunde liegen ist instruktiv und vielversprechend (u. a. Kannengießer, 2019, 2020; Suchmann 2002). So überzeugend die jeweiligen Plädoyers für eine Überwindung des Denkens in ontologischen Entitäten, des Erkennens in dualistischen Weltbildern auch ist, in sozialtheoretischer, -analytischer und insbesondere -kritischer Hinsicht ist ihr Mehrwert bislang überschaubar.

Für die theoretische Perspektive auf Kritik stellt diese Skizze gegenwartsbezogener epistemologischer Diskussionen selbstredend nur einen schmalen Ausschnitt dar. Mittels theoretischer Arbeit werden auch weiterführende Annahmen, Perspektiven und Normen definiert, die es ermöglichen, Kritik wesentlich feinkörniger, konkret phänomen- und problembezogen zu adressieren. Marian Adolf taucht in seinem Artikel in diesem Sonderheft tief in die Geschichte der Definition und Theoretisierung von Kritik ein, beginnend mit Kants Verständnis von Kritik als Urteil, weiterführend mit Marx' und später Horkheimers Kritik an der politischen Ökonomie sowie Foucaults Überlegungen zu Wissen und Macht. Basierend auf einer kurzen Ideengeschichte der Kritik und unter Rückgriff auf Argumente Karl Poppers, konzipiert er Kritik als eine Grundform menschlicher Sozialität und Kommunikation, deren zentrale Funktion es ist, zu zeigen, dass ein anderes Leben möglich ist (Adolf, 2021). In einer Gegenwart von Netzwerk-, Wissens- oder Mediengesellschaften oder hybrider Mediensysteme (Chadwick, 2013) ist, so die Argumentation Adolfs, Sozial- und Gesellschaftskritik daher immer auch mit Medienkritik verbunden. Folglich hat die Medien- und Kommunikationswissenschaft nicht nur die Verantwortung, kritische Theorien auf medienvermittelte und medienbezogene Phänomene und Prozesse anzuwenden, sondern die Pflicht, Medienkritik (bezogen auf Medieninhalte wie -praktiken) als disziplinäre Aufgabe zu begreifen und als grundlegendes Erkenntnisziel zu formulieren.

\section{Kritik als Forschungsgegenstand in der Kommunikations- und Medienwissenschaft}

Schon die frühe Kommunikations- und Medienforschung hat ihre Gegenstände aus einer kritischen Perspektive untersucht (z. B. Horkheimer \& Adorno, 2013 [1944] zur Kulturindustrie). Diese Tradition setzt die Kommunikations- und Medienwissenschaft fort, indem sie kommunikations- und medienbezogene Phänomene und Prozesse im Sinne Kants kritisiert, also beurteilt (z. B. Ang, 1990 explizit kritisch zu Mediensystemen; Downing, 2008 zu alternativen Medien; Fuchs \& Sandoval, 2014 kritisch zur Informationsgesellschaft). 
Aber Kritik war und ist auch selbst Gegenstand der Kommunikations- und Medienforschung, wobei der Fokus hier vor allem auf Kritik als Thema in den Medieninhalten lag und liegt und analysiert wird, wie Medien in ihren Inhalten Medien selbst kritisieren (Hallenberger \& Nieland, 2005; Bucher, 2000). Hartmut Weßler fragt: „Wann ist Medienkritik kritisch?“ und weist darauf hin, dass Medienkritiker*innen und Mediennutzer*innen oftmals die „soziale Neugier“ fehlt, „was man mit Medien alles machen kann (unterschiedliche soziale Gebrauchsweisen) und was man noch alles mit Medien machen kann (kreative Nutzungsmöglichkeiten)“(Weßler, 1997, S. 17, Hervorhebung im Original). Die Autorinnen unseres Sonderhefts beobachten durchaus solche kreativen und alternativen Nutzungsweisen von Medien (Kannengießer \& Möller 2021), und solche sind sicherlich mit Etablierung eines jeden Mediums entwickelt worden (auch in den 1990er Jahren, in denen Weßler diese Frage stellte). Kannengießer und Möller kritisieren aber die Kommunikations- und Medienwissenschaft dafür, einen verkürzten Blick auf Medienkritik als Kritik von Medien an Medien in ihren Inhalten zu werfen (Kannengießer \& Möller, 2021). Die Autorinnen entwickeln den Begriff der kritischen Medienpraktiken und charakterisieren diese durch zwei zentrale Merkmale:

„1) In kritischen Medienpraktiken reflektieren Akteure Routinen, die sich auf Medien (als Organisationen, Inhalte oder Technologien) beziehen, und/ oder Metaprozesse wie Mediatisierung, Digitalisierung oder Datafizierung. 2) Auf der Grundlage dieser Reflektion entwickeln Akteure alternative Routinen in ihren Medienpraktiken und gestalten Prozesse der Mediatisierung, Digitalisierung oder Datafizierung.“ (Kannengießer \& Möller, 2021, 256).

Sie argumentieren, dass die Kommunikations- und Medienwissenschaft auch verschiedene Modi kritischer Medienpraktiken analysieren muss, um Digitalisierungsund Datafizierungsprozesse besser zu verstehen und zu begreifen wie verschiedene Akteur*innen (Individuen, Nichtregierungsorganisationen und Unternehmen) diese Prozesse kritisieren und gestalten. Damit fordern sie das Fach auf, den Blick zu weiten und Kritik nicht nur in Medieninhalten wahrzunehmen, sondern auch in Medienpraktiken.

\section{Kritik und empirische Methoden}

Die empirische kommunikationswissenschaftliche Analyse ist idealtypisch mit einer kritischen Reflexion der angewandten Methoden und der verwendeten Datenquellen verknüpft. Dies kann sich ganz grundlegend auf die bereits erwähnte kritische Reflexion der Verwendung von Big Data beziehen, und bedeutet ebenso grundlegend eine permanente (Selbst-)Kritik des gesamten empirischen Prozesses, von der Qualität der Operationalisierung, über die Datenerhebung bis zur Datenauswertung. Die Standardisierung der empirischen Methoden vereinfacht eine solche Kritik. Mitunter führt dies auch zu einer Methodenkritik als eine von den eigentlichen inhaltlichen Fragen losgelösten Kritik, was auch wiederum kritisch hinterfragt werden kann.

Eine Kritik an Methoden und Daten kann und soll sich mit den Maßstäben, nach denen Daten bewertet und als valide eingestuft werden, befassen, ebenso wie 
mit notwendigen pragmatischen Abwägungen aus forschungsökonomischen Gründen, die Beschränkungen der empirischen Arbeit erfordern. Welche Daten sammeln wir, und welche Daten sollten wir sammeln, um zu validen Erklärungen sozialer Phänomene zu kommen, das ist die grundlegende Frage der Kritik an Methoden. Weiterführend stellt sich die Frage, wen wir ausschließen aus unseren Forschungsergebnissen, weil bestimmte Methoden der Datenerhebung gewählt werden (etwa bei Online-Erhebungen, die Nicht-Nutzer*innen des Internets nicht berücksichtigen können). Noch ein Schritt weiter kann die Wahl der Methode auch als impliziter Ausdruck von Macht betrachtet werden, durch die eine Reproduktion bestehender Ungleichheiten erfolgt - und wodurch die Ergebnisse empirischer Arbeiten letztlich nur bedingt aussagekräftig und verallgemeinerbar sind (Henrich et al., 2010) oder kulturelle Vielfalt unzureichend berücksichtigt wird (Broesch et al., 2020).

Die möglichen Probleme bei der Verwendung von nicht-wissenschaftlichen Daten in kommunikationswissenschaftlichen Arbeiten reflektiert Silke Fürst (2021) in ihrem Beitrag in diesem Band. Fürst benennt als eine wesentliche Problemstelle die unhinterfragte Übernahme von Daten und Metriken, die von kommerziellen Akteur"innen generiert werden, und die dann mitunter nicht kontextualisiert weiterverwendet werden und deren methodischer Hintergrund nicht hinterfragt wird. Aus einem anderen Blickwinkel betrachtet kann Kritik auch eine Methode sein, wie Marian Adolf (2021) in seinem Beitrag ausführt.

\section{Die Relevanz von Kritik in der Kommunikationswissenschaft}

Fürsts Überlegungen, welche Daten wir in der Kommunikations- und Medienforschung eigentlich sammeln und analysieren, führen auch zu einer breiteren Reflexion über die abnehmende Relevanz von Kritik in unserer Disziplin. Zwei Beiträge in diesem Sonderheft stellen deutlich das Dilemma heraus, dass die Disziplin der Kommunikationswissenschaft ihre kritischen Perspektiven teilweise verloren hat. Friedrich Krotz (2021) skizziert in seinem Beitrag die Vielfalt und Geschichte kritischer Zugänge, Tagungen und Projekte in der Kommunikationswissenschaft. Allerdings muss diese Haltung in Zeiten der Digitalisierung nicht nur gestärkt, sondern überdacht werden. Er fordert daher am Beispiel der Entstehung und Entwicklung des Computers als zentrales Medium menschlichen Kommunizierens eine kritische Prozessforschung. Diese Form kritischer Analyse vergleicht die möglichen mit den existierenden Mediennutzungsformen. Durch diese Kritik am Bestehenden wird es möglich, Räume für Utopie(n) zu schaffen.

Klaus-Dieter Altmeppen (2021) vertieft in seinem Beitrag den Blick auf die öffentliche Resonanz der Kommunikationswissenschaft, indem er pointiert Ursachen und Folgen der offensichtlichen Sprachlosigkeit der Kommunikationswissenschaft im öffentlichen Diskurs benennt. Kommunikationswissenschaft muss dabei stetig sich selbst kritisch reflektieren, um nicht nur ein guter Ratgeber für die Öffentlichkeit sein zu können, sondern emanzipatorisch in sie hineinzuwirken. In Zeiten der Radikalisierung politischer Diskurse ist es nicht nur angebracht, sondern zwingend notwendig, dass unsere Disziplin ihre Komfortzone verlässt.

Worauf ist es aber zurückzuführen, dass sich eine kritische Perspektive in der Kommunikationswissenschaft nicht nur früher, sondern auch gegenwärtig mehr 
denn je so schwertut? Michael Meyen (2019) verweist unter anderem auf die fehlende gesellschaftstheoretische Rahmung einer Vielzahl von Studien und Perspektiven und deren aus kritischer Perspektive zu eng gedachter Fokusse auf Theorien mittlerer Reichweite und damit einhergehender Methodologien. Allerdings sind gerade in den letzten Jahren eine Vielzahl von kritischen Perspektiven artikuliert worden. Um nur ein Beispiel zu nennen: Die intensive Kritik an der theoretischen Konzeptionierung und empirischen Fassung des zentralen Formalobjekts unserer Disziplin, die „öffentliche Kommunikation“ (Hepp et al., 2016, Schäfer \& Weßler, 2020). Gerade in der Vielzahl kritischer Ansätze in jüngster Zeit liegt ein Gewinn, nämlich die Möglichkeit der Etablierung eines heterogenen und emanzipierenden kritischen Diskurses. Allerdings lässt das Ausmaß der Argumente und Befunde den gegenwärtigen Zustand der Kritik krisenhaft erscheinen. So gibt es im deutschsprachigen Raum wenige resonanzstarke Analysen, durch die verschiedene Beobachtungen integriert, unterschiedliche Ursachen benannt und schlüssig synthetisiert werden.

Neben den theoretischen wie empirischen Perspektiven ist auch die Form der Wissensproduktion kritisch zu reflektieren, und die ist weitaus schwieriger zu reformieren. So erscheinen die strukturellen Rahmenbedingungen und die Arbeitsprozesse des universitären und wissenschaftlichen Betriebes in Zeiten einer auch in der Wissenschaft zum Teil auffindbaren „Ellenbogengesellschaft“ kritischen Perspektiven abträglich, eine rationale Wissensproduktion gegenüber einer normativen gar karrierefördernder. Das spiegelt sich auch in der Forschungsförderung und den Ressourcen in und zur Forschung wider. Und sicherlich fördern auch die Bedingungen der Qualifikationsprozesse im deutschen Wissenschaftssystem und insbesondere das Wissenschaftszeitvertragsgesetz nicht die Ausbildung von Kritikfähigkeit und Möglichkeiten der Kritikartikulation junger Wissenschaftler*innen. Diese Umstände sowie fehlende Debatten über das Menschen- und Gesellschaftsbild der Kommunikationswissenschaft können - so unsere Befürchtung - die Haltung von Wissenschaftler*innen nachhaltig prägen. Es steht daher nun an, die Privilegien und Positionierungen der Forscher*innen sowie Prozesse der Inklusion und Exklusion in der bestehenden Wissensproduktion kritisch zu analysieren.

\section{Forschungslücken und offene Fragen}

Die Beiträge dieses Sonderheftes liefern wertvolle Schlaglichter auf die unterschiedlichen Facetten von Kritik hinsichtlich Theorien, Forschungsgegenständen, Methoden und dem disziplinären Selbstverständnis der Kommunikationswissenschaft. Das Sonderheft trägt viele relevante Aspekte und Fallbeispiele zusammen, die in den Beiträgen thematisiert und analysiert werden. Diese Schlaglichter zeigen aber auch noch mehr Fragen und Forschungsdesiderate auf: Welche Rolle, zum Beispiel, spielen die verschiedenen Formen von Kritik in der digitalen und datafizierten Gesellschaft? Wie ist das Verhältnis von Medienkritik und Gesellschaftskritik? Welche öffentlichen Praktiken, Diskurse und Öffentlichkeiten prägen Medienkritik und Kritik an der gesellschaftlichen Rolle der Medien? Welche Konsequenzen ergeben sich aus den Erkenntnissen der Kritik an Theorien, Forschungszielen und Methoden für die Medienforschung, die Medienpolitik, den Journalismus oder die Medienpädagogik? 
In Zeiten dramatischer Umbrüche muss sich die Kommunikationswissenschaft mehr denn je ihrer Verantwortung stellen, die Relevanz und Kraft von Kritik neu zu beleben.

\section{Literatur}

Adolf, M. (2021). Kritik als Aufgabe. Zum Kritikbegriff der Sozial- und Kulturwissenschaften [Criticism as a task. On the concept of criticism in the social and cultural sciences]. Studies in Communication and Media, 10(2), 146-167. https://doi.org/10.5771/2192-4007-2021-2-146

Altmeppen, K-D. (2021). Mittendrin und trotzdem nicht dabei. Zugespitzte Anmerkungen zur nichts sagenden Bedeutung der Kommunikationswissenschaft in der Gesellschaft [Right in the middle and still not there. Pointed remarks on the meaningless importance of communication science in society]. Studies in Communication and Media, 10(2), 269-283. https://doi.org/10.5771/2192-4007-2021-2-268

Anderson, C. (2008). The end of theory: The data deluge makes the scientific method obsolete. https://www.wired.com/2008/06/pb-theory/

Ang, I. (1990). Culture and communication: Towards an ethnographic critique of media consumption in the transnational media system. European Journal of Communication, 5(2), 239-260.

Broesch, T., Broesch, T., Crittenden, A. N., Beheim, B. A., Blackwell, A. D., Bunce, J. A., Colleran, H., Hagel, K., Kline, M., McElreath, R., Nelson, R. G., Pisor, A. C., Prall, S., Pretelli, I., Purzycki, B., Quinn, E. A., Ross, C., Scelza, B., Starkweather, K., Stieglitz, J., \& Borgerhoff Mulder, K. (2020). Navigating cross-cultural research: Methodological and ethical considerations. PsyArXiv, June 10. https://doi.org/10.31234/osf.io/thqsw

Bucher, H.-J. (Hrsg.) (2000). Medienkritik zwischen ideologischer Instrumentalisierung und kritischer Aufklärung [Media criticism between ideological instrumentalization and critical education]. Herbert von Halem.

Burkart, R. (2002). Kommunikationswissenschaft. Grundlagen und Problemfelder, Umrisse einer interdisziplinären Sozialwissenschaft [Communication science. Basics and problem areas, outlines of an interdisciplinary social science]. Böhlau.

Chadwick, A. (2013). The hybrid media system. Politics and power. Oxford University Press.

Couldry, N. (2020). Recovering critique in an age of datafication. New Media and Society, 22(7), 1135-1151. https://doi.org/10.1177/1461444820912536

Cukier, K., \& Mayer-Schoenberger, V. (2013). The rise of big data: How it's changing the way we think about the world. Foreign Affairs, 92(3), 28-40. https://doi. org/10.1515/9781400865307-003

Downing, J. (2008). Social movement theories and alternative media: An evaluation and critique. Communication, Culture and Critique, 1(1), 40-50. https://doi. org/10.1111/j.1753-9137.2007.00005.x

Fuchs, C., \& Sandoval M. (Hrsg.). (2014). Critique, social media, and the information society. New York: Routledge.

Fürst, S. (2021). Verstrickt in der Rhetorik der Technologie-, Daten- und Medienunternehmen? Zu Problematiken im Umgang mit nicht-wissenschaftlichen Nutzungsdaten [Caught up in the rhetoric of technology, data, and media companies? Problem- 
atic practices in dealing with nonscientific usage data]. Studies in Communication and Media, 10(2), 175-209. https://doi.org/10.5771/2192-4007-2021-2-175

Gentzel, P (2019). Materialität, Technik und das Subjekt: Elemente kritischer Kommunikations- und Medienanalyse [Materiality, technology and the subject: Elements of critical communication and media analysis]. In P. Gentzel, F. Krotz, J. Wimmer, \& R. Winter (Hrsg.), Das vergessene Subjekt. Subjektkonstitutionen in mediatisierten Alltagswelten (S. 87-113). Springer VS.

Görland, S. (2020). Medien, Zeit und Beschleunigung: Mobile Mediennutzung in Interimszeiten [Media, time and acceleration: Mobile media use in interim periods]. Springer VS.

Hall, S. (1997). Representation. Cultural representation and signifying practices. Sage.

Hallenberger, G., \& Nieland, J.-U. (Hrsg.). (2005). Neue Kritik der Medienkritik. Werkanalyse, Nutzerservice, Sales Promotion oder Kulturkritik? [New criticism of media criticism. Work analysis, user service, sales promotion or cultural criticism?] Herbert von Halem.

Heidegger, M. (2000). Die Frage nach der Technik [The question of technology]. In Gesamtausgabe Band 7: Vorträge und Aufsätze (S. 5-36). Klostermann.

Henrich, J., Heine, S. J., Norenzayan, A. (2010). The weirdest people in the world? Behavioral and Brain Sciences, 33(2-3), 61-83. https://doi.org/10.1017/S0140525X0999152X

Horkeimer, M., \& Adorno, T. W. (2013 [1944]). Dialektik der Aufklärung [Dialectic of Enlightenment] (21. Aufl.). Fischer.

Kallmeyer. M. (2017). New Materialism: neue Materialitätskonzepte für die Gender Studies [New Materialism: New materiality concepts for gender studies]. In B. Kortendiek, B. Riegraf, \& K. Sabisch (Hrsg.), Handbuch Interdisziplinäre Geschlechterforschung, Geschlecht und Gesellschaft (S. 1-10). Springer.

Kalthoff, H. (2019). Materieller Sinn. Die Soziologie der Materialität und die Daseinsweisen der Dinge [Material meaning. The sociology of materiality and the way things exist]. Zeitschrift für theoretische Soziologie, 8(2), 147-172.

Kannengießer, S. (2019). Reflecting and acting on datafication. CryptoParties as an example of critical media practices. Convergence: The International Journal of Research into New Media Technologies. https://doi.org/10.1177/1354856519893357

Kannengießer, S. (2020). Fair media technologies: innovative media devices for social change. The Journal of Media Innovations, 6(1), 38-49. https://doi.org/10.5617/ jomi.7832

Kannengießer, S., \& Möller, J. E. (2021). Critical Media Practices. Studies in Communication and Media, 10(2), 254-268. https://doi.org/10.5771/2192-4007-2021-2-253

Krallmann, D., \& Ziemann, A. (2001). Grundkurs Kommunikationswissenschaft [Communication science basic course]. Fink.

Krotz, F. (2008). Sind Medien Kanäle? Ist Kommunikation Informationstransport? Das mathematisch/technische Kommunikationsmodell und die sozialwissenschaftliche Kommunikationsforschung [Are media channels? Is communication the transport of information? The mathematical / technical communication model and social science communication research]. In K.-S. Rehberg (Hrsg.), Die Natur der Gesellschaft: Verhandlungen des 33. Kongresses der Deutschen Gesellschaft für Soziologie in Kassel 2006 (S. 1044-1059). Campus.

Krotz, F. (2021). Das Mögliche als Kritik des Existierenden: Kritische Forschung in der Kommunikationswissenschaft und ein Beispiel kritischer Prozessforschung über die En- 
tstehung und Entwicklung des Computers [The possible as a critique of the existing: Critical research in communication science and an example of critical process research on the origin and development of the computer]. Studies in Communication and Media, 10(2), 223-246. https://doi.org/10.5771/2192-4007-2021-2-222

Latour, B. (2004). Why has critique run out of steam? From matters of fact to matters of concern. Critical Inquiry, 30, 225-248. https://doi.org/10.1086/421123

Luckner, A. (2008). Heidegger und das Denken der Technik [Heidegger and the thinking of technology]. transcript.

Manovich, L. (2020). Cultural analytics. MIT Press.

Meyen, M. (2019). Die Definitionsmacht der Kommunikationswissenschaft. Ein Plädoyer für eine „wissenschaftsgeschichtliche Besinnungspause“ und eine Replik auf „Woher kommt und wozu führt Medienfeindlichkeit? “ [The power of definition of communication science. A plea for a "pause for reflection on the history of science" and a reply to "Where does media hostility come from and what does it lead to?"] Medien \& Kommunikationswissenschaft, 67(1), 77-87. https://doi.org/10.5771/1615-634X-2019-1-77

Neverla, I. (1992). Fernseh-Zeit. Zuschauer zwischen Zeitkalkül und Zeitvertreib [TV time. Spectators between time calculation and pastime]. Ölschläger.

Pürer, H. (2003). Publizistik und Kommunikationswissenschaft. Ein Handbuch [Spaces and contexts of public communication]. UVK.

Savage, M (2015). The new public social science of 'big data'. Keynote Dialogue in the series "making sense of big data". Alexander von Humboldt Institut für Internet und Gesellschaft. Berlin. Abgerufen von https://www.youtube.com/watch?v=fZkJh0pfAUE \&list=PLXMgjqxV5HdJBfwETXVGoUpNjAFFZ_1xM\&index=7

Schatzki, T. R. (2001). Introduction. In T.R. Schatzki, K. Knorr-Cetina, E. v. Savigny

(Hrsg.), The practice turn in contemporary theory (S. 1-14). Routledge.

Schatzki, T. R., Knoor-Cetina, K., \& von Savigny, E. (2001). The practice turn in contemporary theory. Routledge.

Suchman, L. A. (2002). Practice-based design of information systems: Notes from the hyperdeveloped world. The Information Society, 18, 139-144.

Wallner, C, \& Adolf, M. (2014). Räume und Kontexte öffentlicher Kommunikation [Spaces and contexts of public communication]. In J. Wimmer \& M. Hartmann (Hrsg.), Medienkommunikation in Bewegung. Mobilisierung - Mobile Medien Kommunikative Mobilität (S. 87-101). Springer VS.

Weber, M. (1988 [1922]). Wirtschaft und Gesellschaft. Grundriß der verstehenden Soziologie [Economy and society. Outline of understanding sociology]. Mohr.

Weßler, H. (1997). Der ,befremdete Blick' auf das Selbstverständliche. Wann ist Medienkritik kritisch? [The 'alienated view' of the obvious. When is media criticism critical?] In H. Weßler, C. Matzen, O. Jarren, \& U. Hasebrink (Hrsg.), Perspektiven der Medienkritik. Die gesellschaftliche Auseinandersetzung mit öffentlicher Kommunikation in der Mediengesellschaft (S. 15-26). Westdeutscher Verlag.

Zuboff, S. (2019). The age of surveillance capitalism. The fight for a buman future at the new frontier of power. Profile. 